\title{
Case $-{ }^{18}$ F-DCFPyL-positron emission tomography/computed tomography (PET/CT) time of imaging
}

Golmehr Sistani, MD'; Ur Metser, MD²; Glenn S. Bauman, $\mathrm{MD}^{3}$; David T. Laidley, $\mathrm{MD}^{4}$; Stephen E. Pautler, MD ${ }^{5}$; Katherine A. Zukotynski, MD, $\mathrm{PhD}^{6}$

${ }^{1}$ Department of Medical Imaging, Western University, London, ON, Canada; ${ }^{2}$ Department of Radiology, University of Toronto, Toronto, ON, Canada; ${ }^{3}$ Department of Radiation Oncology, Western University, London, ON, Canada; ${ }^{4}$ Department of Nuclear Medicine, Western University, London, ON, Canada; ${ }^{5}$ Department of Surgery, Division of Urology, Western University, London, ON, Canada; ${ }^{6}$ Department of Medicine and Radiology, McMaster University, Hamilton, ON, Canada

Cite as: Sistani G, Metser U, Bauman GS, et al. Can Urol Assoc J 2020 December 15; Epub ahead of print. http://dx.doi.org/10.5489/cuaj.6984

Published online December 15, 2020

$* * *$

\section{Introduction}

Prostate cancer is the most commonly diagnosed cancer, except for skin cancer, and the second leading cause of cancer related death among Canadian males. ${ }^{1}$ Recurrence is not uncommon, despite local therapies such as radical prostatectomy, radiation and brachytherapy and systemic therapies including hormonal therapy. ${ }^{2}$ Often, the first sign of recurrence is a rising PSA, known as "biochemical recurrence". ${ }^{3}$ Based on the European Association of Urology and American Urological Association guidelines, biochemical recurrence is defined as serum PSA value of $\geq$ $0.2 \mathrm{ng} / \mathrm{ml}$ after prostatectomy and $\geq 2 \mathrm{ng} / \mathrm{ml}$ increase above the nadir PSA after external beam radiation therapy of the primary tumour. ${ }^{3}$ In recent years, prostate specific membrane antigen targeted positron emission tomography/ computed tomography (PSMA-PET/CT) has revolutionized detection of disease in men with biochemical recurrence and has led to a change in management. ${ }^{4}$

${ }^{68}$ Ga-PSMA-11 is arguably the most widely used PET radiopharmaceutical world-wide for the detection of prostate cancer. However, it has limitations such as short physical half-life (68 min). ${ }^{18}$ F-labeled PSMA-targeting radiopharmaceutical are easier to distribute given their longer half-life (110 minutes). ${ }^{18} \mathrm{~F}$-DCFPyL is the most commonly used ${ }^{18} \mathrm{~F}$-labeled PSMAtargeting radiopharmaceutical in Canada. The biodistribution patterns of ${ }^{68} \mathrm{Ga}$-PSMA and ${ }^{18} \mathrm{~F}$ DCFPyL are similar. ${ }^{5}$ Many centers acquire images 60 minutes post intravenous administration of ${ }^{18} \mathrm{~F}$-DCFPyL. In this paper we illustrate the value of dual-time point imaging and compare the 
imaging at 60 and 120 minutes post radiopharmaceutical injection. All cases had biochemical recurrence with negative bone scan and CT scan.

\section{Case 1}

70-year-old man with biochemically recurrent prostate cancer (PSA $0.3 \mathrm{ng} / \mathrm{mL}$ ) was referred for PSMA-PET/CT. He was treated with radical prostatectomy 10 years ago and hormonal therapy and has been off of hormonal therapy 2 years ago. The creatinine was $78 \mu \mathrm{mol} / \mathrm{L}$. Total body images obtained 60 minutes following intraveous ${ }^{18} \mathrm{~F}$-DCFPyL administration showed intense focal radiopharmaceutical uptake in a left internal iliac lymph node and mild focal uptake in para-aortic soft tissue to the left of the aortic bifurcation (Figure 1A). Additional imaging of the pelvis performed 120-minutes post radiopharmaceutical injection, showed interval increase uptake in the left internal iliac lymph node consistent with prostate cancer spread and interval decrease in uptake in the para-aortic soft tissue consistent with the organ of Zuckerkandl (Figure 1B). Radiopharmaceutical uptake in the sympathetic ganglia and organ of Zuckerkandl can be mistaken for malignant disease spread and is a known pitfall of PSMA-PET/CT. ${ }^{6}$ In addition to the lesion location and shape, interval reduction in conspicuity on delayed images suggests a benign process, while interval increase in conspicuity suggests malignant disease spread. ${ }^{6,7}$ This case illustrates the value of dual time-point ${ }^{18} \mathrm{~F}$-DCFPyL PET/CT, similar to multi-phase ${ }^{68} \mathrm{Ga}$ PSMA PET/CT. ${ }^{8}$ In this case, the staging could have been potentially changed as internal iliac node (the shown avid node) is a locoregional node for prostate cancer but a node at the level of aortic bifurcation is considered metastatic.

\section{Case 2}

69-year-old man with biochemically recurrent prostate cancer (PSA $10.9 \mathrm{ng} / \mathrm{mL}$ ) 3 years post external radiation therapy, was referred for PSMA-PET/CT. The creatinine was $74 \mu \mathrm{mol} / \mathrm{L}$. The images obtained 60 minutes following intravenous ${ }^{18} \mathrm{~F}$-DCFPyL administration showed equivocal mild focal uptake in a prevertebral soft tissue nodule (Figure 2A). Interval increase in the radiopharmaceutical uptake on delayed images obtained 120 minutes following intravenous

${ }^{18} \mathrm{~F}$-DCFPyL administration, however, was consistent with prostate cancer spread to a small presacral lymph node (Figure 2B). Given high PSA at biochemical recurrence, hormone therapy was commenced. Also, the patient was offered regional pelvic radiation therapy given the positive PSMA PET/CT finding. His PSA dropped to $1.02 \mathrm{ng} / \mathrm{mL}$ post treatment.

\section{Case 3}

77-year-old man with history of Gleason $4+3$ prostate adenocarcinoma developed biochemically recurrent prostate cancer 8 years post radical prostatectomy (PSA $1.7 \mathrm{ng} / \mathrm{mL}$ ) and subsequently was referred for PSMA-PET/CT. The creatinine was $130 \mu \mathrm{mol} / \mathrm{L}$. The images obtained 60 minutes following radiopharmaceutical administration showed intense focal radiopharmaceutical uptake in the prostate bed without a suspicious correlating anatomic abnormality, suspicious for 
prostate cancer. Indeed, the retro-vesical region or prostate bed is a common site of local recurrence. ${ }^{9}$ However, this radiopharmaceutical activity resolved on 120 minutes images consistent with physiologic activity in the genitourinary tract (Figures $3 \mathrm{~A}$ and $\mathrm{B}$ ). In addition, a mildly ${ }^{18} \mathrm{~F}$-DCFPyL-avid right obturator node, which may have been missed on early imaging (SUVmax 1.7), showed increasing conspicuity on delayed imaging (SUVmax 2.6) consistent with malignant disease spread. The patient underwent external beam radiation of a single node detected on delayed imaging and his PSA became undetectable.

\section{Case 4}

62-year-old man with biochemically recurrent prostate cancer adenocarcinoma 14 years post radical prostatectomy (PSA was $1.3 \mathrm{ng} / \mathrm{mL}$ ), was referred for PSMA-PET/CT. The creatinine was $78 \mu \mathrm{mol} / \mathrm{L}$. Focal intense radiopharmaceutical uptake in a small peri-ureteric left external iliac lymph node on images obtained 60 minutes following radiopharmaceutical administration, increased in conspicuity at 120 minutes consistent with prostate cancer spread. On the other hand, focal intense uptake in the right peri-ureteric region on images obtained 60 minutes following radiopharmaceutical administration, resolved at 120 minutes consistent with physiologic activity in the genitourinary tract. Repeat delayed imaging in cases of urine activity is helpful to avoid upstaging or downstaging cases.

\section{Discussion}

While the ratio of target-to-background radiopharmaceutical uptake on PSMA-targeted PET/CT typically decreases over time in benign and physiologic processes, it increases in malignant disease, making dual time-point imaging helpful. ${ }^{10,11}$ As illustrated above, benign uptake such as ganglion uptake and urine activity were reduced and cleared on delayed images while involved nodes showed interval increase in conspicuity. Unfortunately, practical considerations including workflow issues and patient preference may limit implementation of dual time-point imaging in clinical practice. ${ }^{7}$ Given issues of accessibility, dual time point imaging could be reserved for characterization of equivocal findings. While checking individual cases prior to a patient leaving the department may be an option for centers with low PSMA PET/CT volume, ultimately, if a choice must be made to perform either early and late imaging, an uptake time of 120 minutes is preferred..$^{10}$ This is true regardless of whether the radiopharmaceutical used for PSMA-targeted PET is labeled with gallium-68 or fluoride- $18 .^{10-14}$ 


\section{References}

1. Brenner DR, Weir HK, Demers AA, Ellison LF, Louzado C, Shaw A, et al. Projected estimates of cancer in Canada in 2020. CMAJ 2020;192(9):199-205.

2. So AI, Chi KN, Danielson B, Fleshner NE, Kapoor A, Niazi T, et al. Canadian Urological Association-Canadian Urologic Oncology Group guideline on metastatic castration-naive and castration-sensitive prostate cancer. Canadian Urological Association Journal 2020;14(2):17.

3. Gillessen S, Attard G, Beer TM, Beltran H, Bjartell A, Bossi A, et al. Management of patients with advanced prostate cancer: report of the Advanced Prostate Cancer Consensus Conference 2019. European urology 2020;77(4):508-547.

4. Perera M, Papa N, Roberts M, Williams M, Udovicich C, Vela I, et al. Gallium-68 prostate-specific membrane antigen positron emission tomography in advanced prostate cancer - updated diagnostic utility, sensitivity, specificity, and distribution of prostatespecific membrane antigen-avid lesions: a systematic review and meta-analysis. European urology 2020;77(4):403-17.

5. Ferreira G, Iravani A, Hofman MS, Hicks RJ. Intra-individual comparison of $68 \mathrm{Ga}-$ PSMA-11 and 18F-DCFPyL normal-organ biodistribution. Cancer Imaging 2019;19(1):23.

6. Rischpler C, Beck TI, Okamoto S, et al. (68)Ga-PSMA-HBED-CC Uptake in Cervical, Celiac, and Sacral Ganglia as an Important Pitfall in Prostate Cancer PET Imaging. $J$ Nucl Med 2018;59(9):1406-1411.

7. Afshar-Oromieh A, Sattler LP, Steiger K, et al. Tracer uptake in mediastinal and paraaortal thoracic lymph nodes as a potential pitfall in image interpretation of PSMA ligand PET/CT. Eur J Nucl Med Mol Imaging 2018;45(7):1179-1187.

8. Beheshti M, Manafi-Farid R, Geinitz H, et al. Multi-phasic (68)Ga-PSMA PET/CT in detection of early recurrence in prostate cancer patients with PSA $<1 \mathrm{ng} / \mathrm{ml}$ : a prospective study of 135 cases. $J$ Nucl Med 2020;61(10):1484-1490.

9. Barbosa FG, Queiroz MA, Nunes RF, et al. Revisiting Prostate Cancer Recurrence with PSMA PET: Atlas of Typical and Atypical Patterns of Spread. Radiographics 2019;39(1):186-212.

10. Wondergem M, van der Zant FM, Knol RJJ, et al. (18)F-DCFPyL PET/CT in the Detection of Prostate Cancer at 60 and 120 Minutes: Detection Rate, Image Quality, Activity Kinetics, and Biodistribution. J Nucl Med 2017;58(11):1797-1804.

11. Hoffmann MA, Buchholz HG, Wieler HJ, Rosar F, Miederer M, Fischer N, et al. DualTime Point [68Ga] Ga-PSMA-11 PET/CT Hybrid Imaging for Staging and Restaging of Prostate Cancer. Cancers 2020;12(10):2788.

12. Derlin T, Weiberg D, von Klot C, et al. (68)Ga-PSMA I\&T PET/CT for assessment of prostate cancer: evaluation of image quality after forced diuresis and delayed imaging. Eur Radiol 2016;26(12): 4345-4353.

13. Schmuck S, Nordlohne S, von Klot CA, et al. Comparison of standard and delayed imaging to improve the detection rate of [(68)Ga]PSMA I\&T PET/CT in patients with biochemical recurrence or prostate-specific antigen persistence after primary therapy for prostate cancer. Eur J Nucl Med Mol Imaging 2017;44(6):960-968. 
14. Hofman MS, Hicks RJ, Maurer T, et al. Prostate-specific Membrane Antigen PET: Clinical Utility in Prostate Cancer, Normal Patterns, Pearls, and Pitfalls. Radiographics 2018;38(1):200-217.

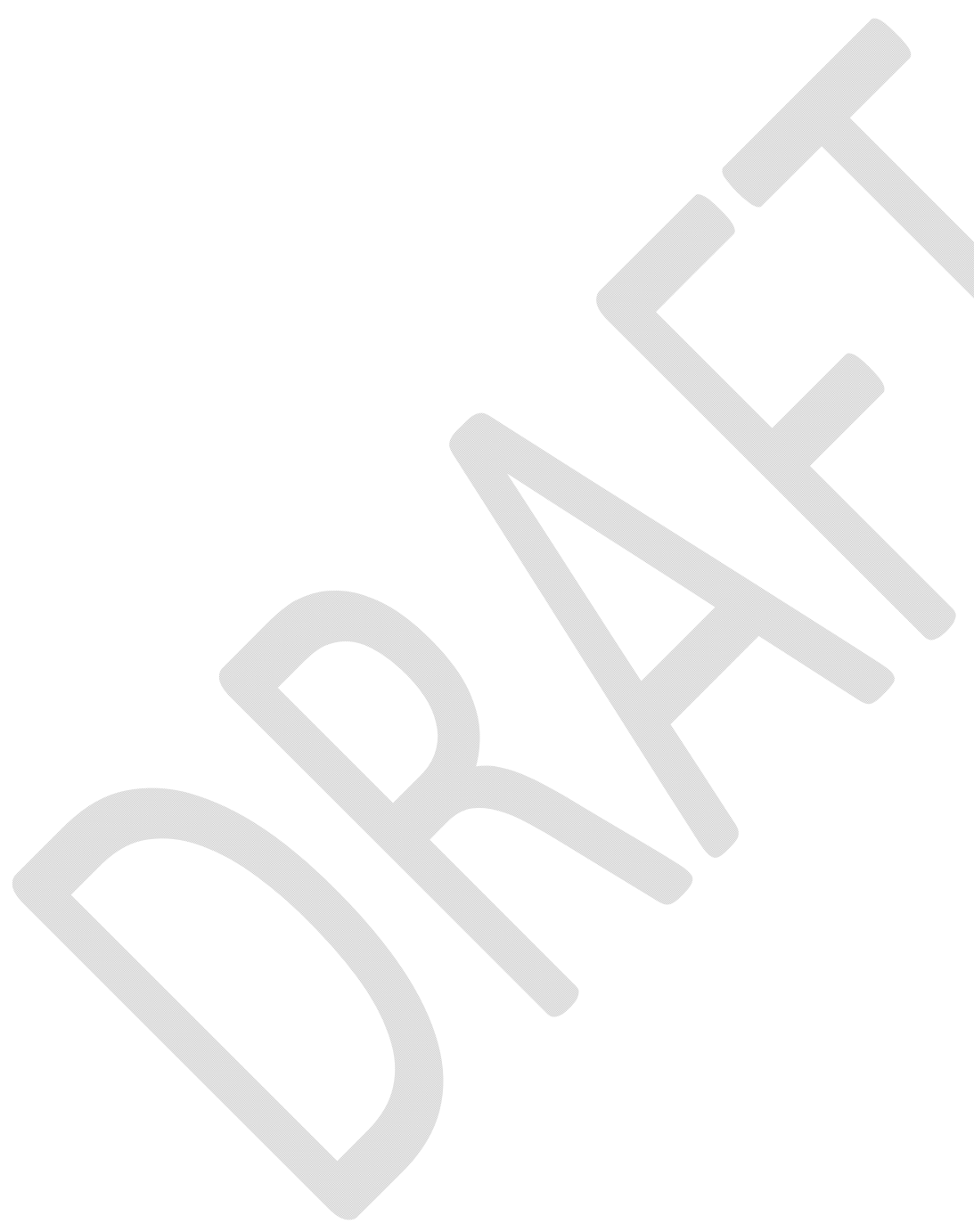




\section{Figures and Tables}

Fig. 1A. Case 1: Total body images obtained 60 minutes following intraveous ${ }^{18} \mathrm{~F}-\mathrm{DCFPyL}$ administration showed intense focal radiopharmaceutical uptake in a left internal iliac lymph node and mild focal uptake in para-aortic soft tissue to the left of the aortic bifurcation.
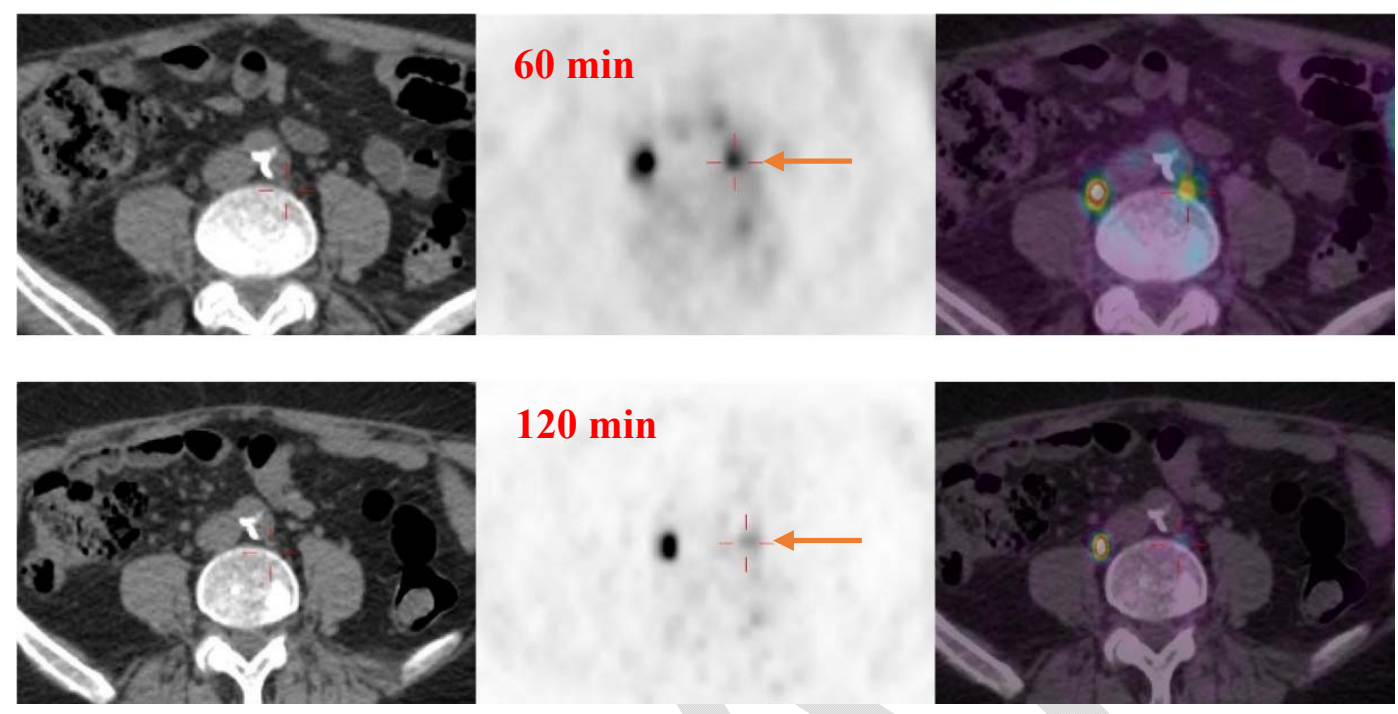

Fig. 1B. Case 1: Additional imaging of the pelvis performed 120 minutes postradiopharmaceutical injection showed interval increase uptake in the left internal iliac lymph node consistent with prostate cancer spread and interval decrease in uptake in the para-aortic soft tissue consistent with the organ of Zuckerkandl.

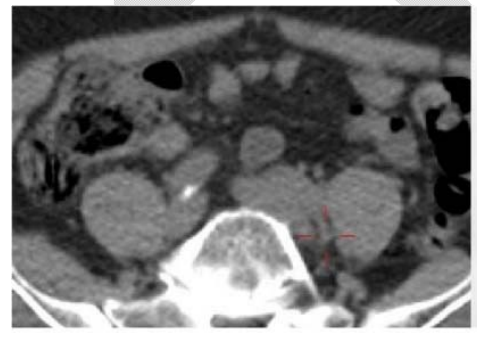

$60 \mathrm{~min}$

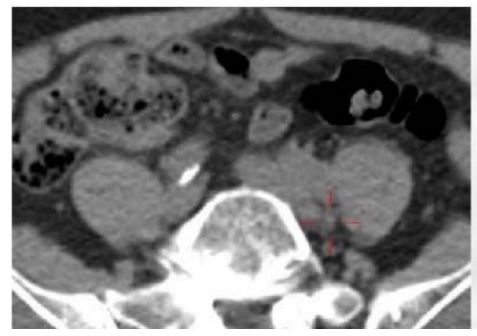

$120 \mathrm{~min}$
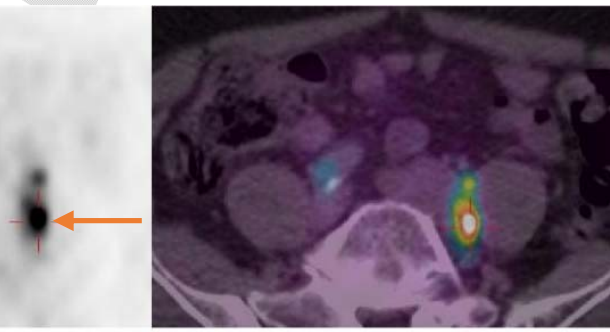
Fig. 2. The images obtained 60 minutes following intravenous ${ }^{18} \mathrm{~F}-\mathrm{DCFPyL}$ administration showed equivocal mild focal uptake in a prevertebral soft tissue nodule (Figure 2A). Interval increase in the radiopharmaceutical uptake on delayed images obtained 120 minutes following intravenous ${ }^{18} \mathrm{~F}$-DCFPyL administration, however, was consistent with prostate cancer spread to a small pre-sacral lymph node (Figure $2 \mathrm{~B}$ )
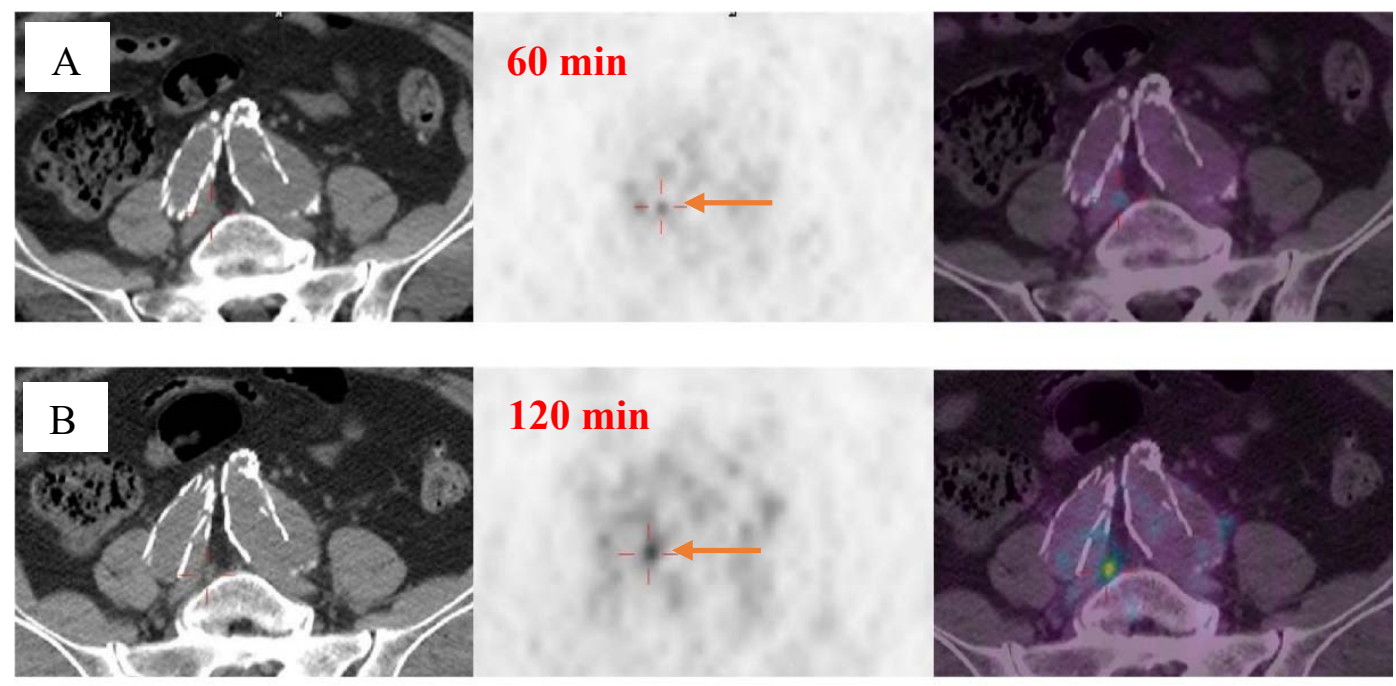

$120 \mathrm{~min}$

Fig. 3. Case 3: The images obtained 60 minutes following radiopharmaceutical administration showed intense focal radiopharmaceutical uptake in the prostate bed without a suspicious correlating anatomic abnormality, suspicious for prostate cancer. However, this radiopharmaceutical activity resolved on 120 minutes images consistent with physiologic activity in the genitourinary tract.

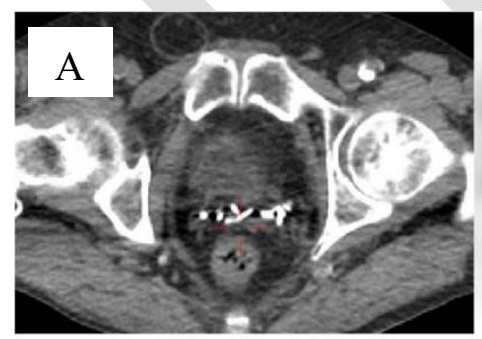

$60 \mathrm{~min}$

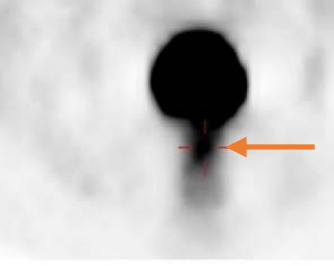

$120 \mathrm{~min}$

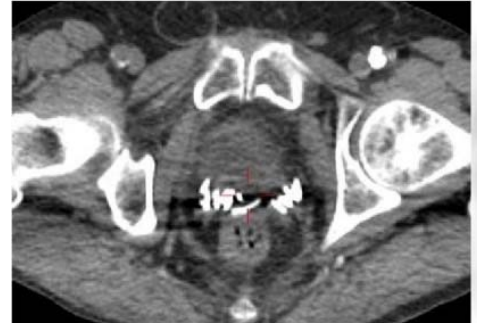

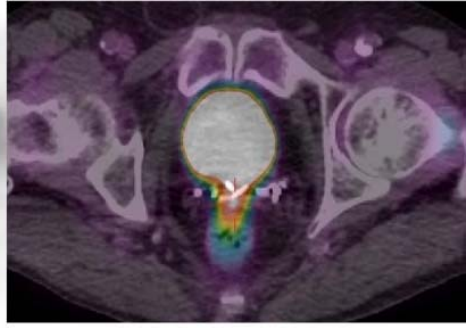

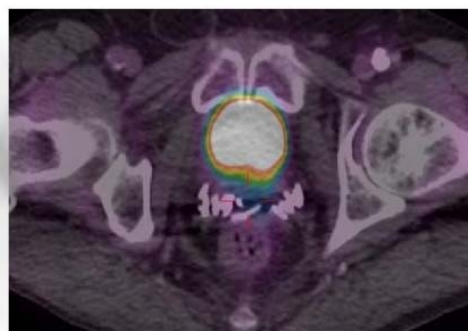



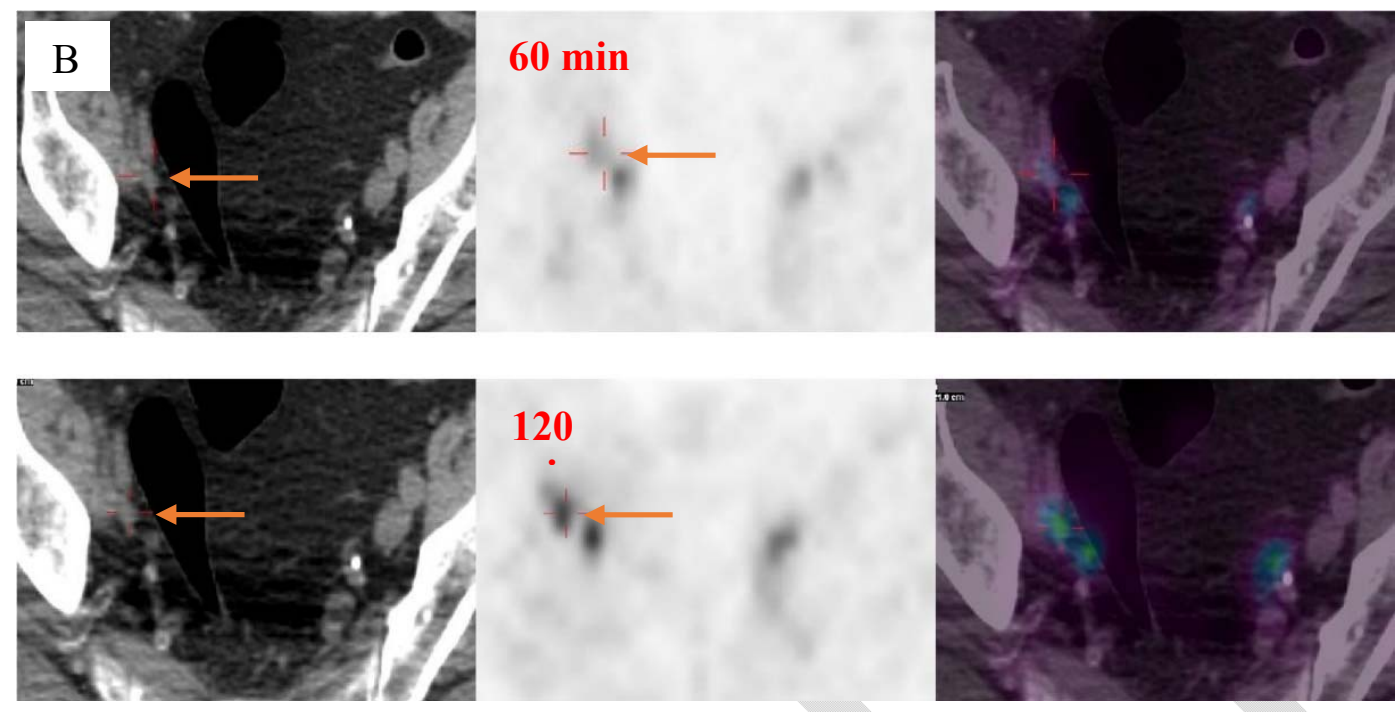

Fig. 4. Case 4: Focal intense radiopharmaceutical uptake in a small peri-ureteric left external iliac lymph node on images obtained 60 minutes following radiopharmaceutical administration, increased in conspicuity at 120 minutes consistent with prostate cancer spread
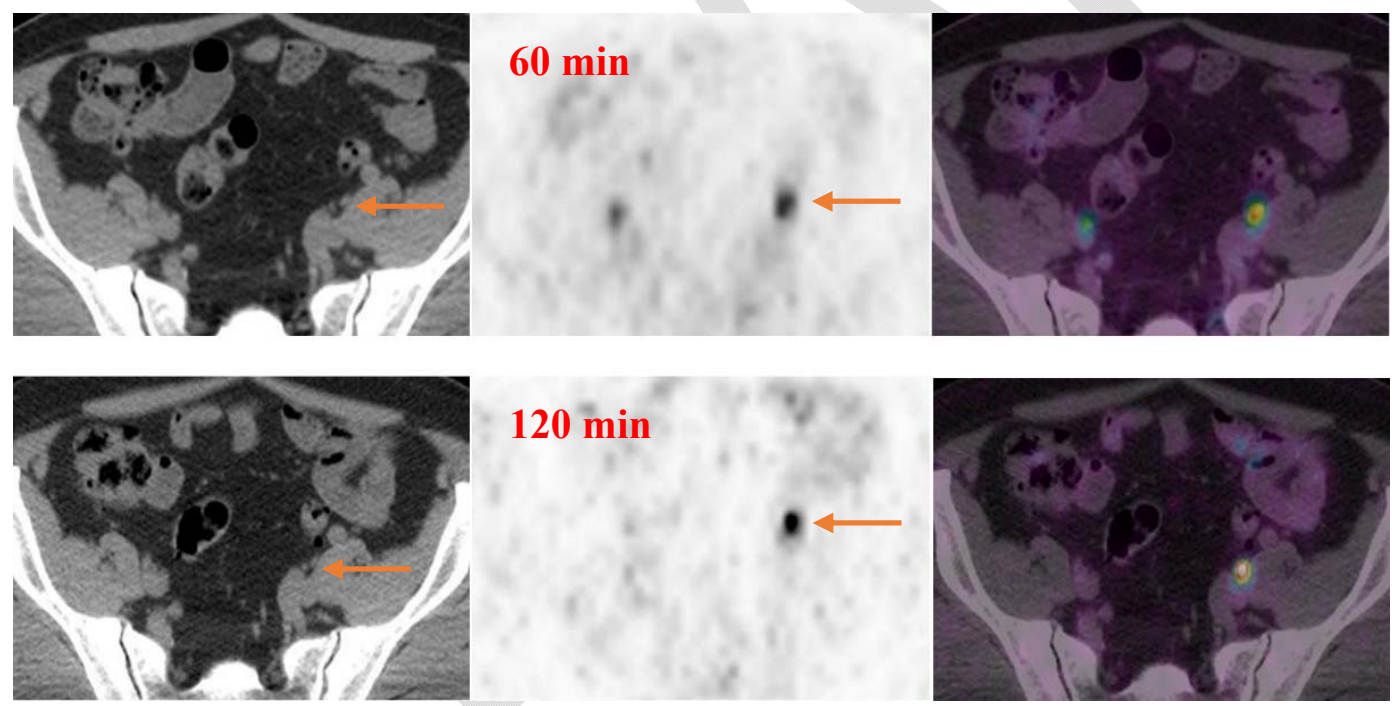

$120 \mathrm{~min}$ 